\title{
TRANSTORNOS ALIMENTARES E INSATISFAÇÃO COM A IMAGEM CORPORAL EM BAILARINOS
}

\author{
EATING DISORDERS AND DISSATISFACTION WITH BODY IMAGE IN BALLET DANCERS \\ TRASTORNOS ALIMENTARIOS E INSATISFACCIÓN CON LA IMAGEN CORPORAL EN BAILARINES
}

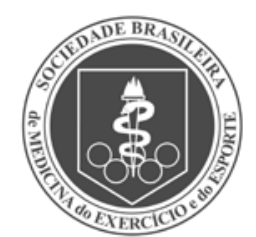

Artigo Original
Aline Dias Guimarães

(Nutricionista)

Soraia Pinheiro Machado² (Nutricionista)

Ana Karina Texeira da Cunha França' (Nutricionista)

Isabela Leal Calado' (Nutricionista)

1. Universidade Federal do Maranhão (UFMA), São Luís, MA, Brasil.

2. Curso de Nutrição da Universidade Estadual do Ceará (UECE), Fortaleza, CE, Brasil.

\section{Correspondência:}

Isabela Leal Calado

Av. dos Portugueses, № 1966.

Cidade Universitária.

Bairro: Bacanga. São Luís, MA.

CEP.65085-580.

belacalado@uol.com.br

\begin{abstract}
RESUMO
Introdução: Os transtornos alimentares (TA) tornaram-se um problema relevante de saúde pública. Paralelamente a esse fato, observa-se aumento na frequência de problemas associados à percepção da imagem corporal. Esses transtornos alimentares são mais incidentes em praticantes de esportes que preconizam o baixo peso corporal, como o balé clássico. Objetivo: Determinar a prevalência de comportamentos de risco de transtornos alimentares e insatisfação com a imagem corporal em bailarinos adolescentes na cidade de São Luís, MA, Brasil. Métodos: Realizou-se estudo transversal com 30 bailarinos clássicos, não profissionais de ambos os sexos, com idade de 15 a 19 anos. Para avaliação do comportamento de risco de anorexia nervosa, utilizou-se o teste de atitudes alimentares (EAT-26), e para o comportamento de risco de bulimia nervosa, o teste de investigação bulímica de Edimburgo (BITE). A imagem corporal foi avaliada por meio do questionário de imagem corporal (BSQ). Resultados: A prevalência de comportamentos de risco de TA foi de 30,0\% para anorexia nervosa, de acordo com o EAT-26 e, segundo a escala de sintomas do BITE, 40,0\% apresentaram comportamento de risco de bulimia nervosa. Além disso, o BSQ identificou que 26,7\% dos bailarinos estavam insatisfeitos com sua imagem. Analisando o comportamento de risco de TA e a insatisfação com a imagem corporal, por sexo, não houve diferença estatisticamente significante $(p>0,05)$. Conclusão: A alta incidência de insatisfação com a imagem corporal associada à grande prevalência de comportamentos de risco de TA encontrada neste estudo ratifica que a prática do balé parece ser um fator primordial para o aumento da suscetibilidade desses bailarinos desenvolverem TA.
\end{abstract}

Palavras-chave: dança, anorexia nervosa, bulimia nervosa, imagem corporal.

\section{ABSTRACT}

Introduction: Eating disorders (ED) have become an important public health problem. Alongside this fact, there is an increase in the frequency of problems associated with the perception of body image. These eating disorders are more incident in sports practitioners who advocate low body weight as classical ballet. Objective: This study aimed to determine the prevalence of risk behaviors of eating disorders and body image dissatisfaction in adolescent ballet dancers in the city of São Luís, MA, Brazil. Methods: A cross-sectional study was conducted with 30 nonprofessional classical ballet dancers of both sexes, aged between 15 and 19 years. To evaluate the risk behavior of anorexia nervosa, we used the eating attitudes test (EAT-26), and the risk behavior of bulimia nervosa, the Bulimic investigatory test, Edinburgh (BITE). Body image was evaluated using the body shape questionnaire (BSQ). Results: The prevalence of risk behaviors for eating disorders was 30.0\% for anorexia nervosa, according to the EAT-26 and, second the BITE symptom scale, $40.0 \%$ had risk behavior of bulimia nervosa. In addition, the BSQ identified that $26.7 \%$ of the dancers were dissatisfied with their image. Analyzing the risk behaviors of eating disorders and the body image dissatisfaction by gender, there was no statistically significant difference ( $p>0.05)$. Conclusion: The high incidence of dissatisfaction with the body image associated with the high prevalence of risk behaviors of ED found in this study confirms that the practice of ballet seems to be a primary factor for the increased susceptibility these ballet dancers to develop eating disorders.

Keywords: dancing, anorexia nervosa, bulimia nervosa, body image.

\section{RESUMEN}

Introducción: Los trastornos alimentarios (TA) se han vuelto un problema relevante de salud pública. Paralelamente a ese hecho, se observa aumento en la frecuencia de problemas asociados a la percepción de la imagen corporal. Esos TA son más incidentes en practicantes de deportes que preconizan el bajo peso corporal, como el balé clásico. Objetivo: Determinar la prevalencia de comportamientos de riesgo de trastornos alimentarios e insatisfacción con la imagen corporal en bailarines adolescentes en la ciudad de São Luís, MA, Brasil. Métodos: Se realizó estudio transversal con 30 bailarines clásicos, no profesionales de ambos sexos, con edad de 15 a 19 años. Para evaluación del comportamiento de riesgo de anorexia nerviosa, se usó el test de actitudes alimentarias (EAT-26), y para el comportamiento de riesgo de bulimia nerviosa, el test de investigación bulímica de Edimburgo (BITE). La imagen corporal fue evaluada por medio del cuestionario de imagen corporal (BSQ). Resultados: La prevalencia de comportamientos de riesgo de TA fue de 30,0\% para anorexia nerviosa, de acuerdo con el EAT-26 y, según la escala de síntomas de 
BITE, 40,0\% presentó comportamiento de riesgo de bulimia nerviosa. Además, el BSQ identificó que 26,7\% de los bailarines estaba insatisfecho con su imagen. Analizando el comportamiento de riesgo de TA y la insatisfacción con la imagen corporal, por sexo, no hubo diferencia estadísticamente significativa $(p>0,05)$. Conclusión: La alta incidencia de insatisfacción con la imagen corporal asociada a la gran prevalencia de comportamientos de riesgo de TA encontrada en este estudio ratifica que la práctica de balé parece ser un factor primordial para el aumento de la susceptibilidad de que esos bailarines desarrollen TA.

Palabras clave: danza, anorexia nerviosa, bulimia nerviosa, imagen corporal.

\section{INTRODUÇÃO}

Os transtornos alimentares (TA) tornaram-se um relevante problema de saúde pública, considerando que a incidência e a prevalência têm aumentado notavelmente nos últimos anos ${ }^{1}$. Paralelamente a este fato, tem-se observado um aumento na frequência de problemas associados à percepção da imagem corporal².

Estes transtornos são definidos como desordens psiquiátricas caracterizadas por distorção da imagem corporal e comportamentos alimentares anormais, causando prejuízos biológicos, psicológicos e aumento da morbimortalidade nos indivíduos ${ }^{3}$. Sua etiologia é de origem multifatorial, na qual inúmeros fatores interagem entre si de modo complexo. Dentre os principais fatores, destacam-se os genéticos, biológicos, psicológicos e socioculturais ${ }^{4}$. Esses transtornos têm início, geralmente na adolescência, acometendo mais mulheres ${ }^{3}$, e o seu diagnóstico é feito por meio dos critérios do Manual Diagnóstico e Estatístico de Transtornos Mentais (DSM- IV) ${ }^{5}$ e da Classificação Internacional de Doenças (CID-10).

Os TA são divididos em duas categorias principais: anorexia nervosa e bulimia nervosa ${ }^{5}$. A anorexia nervosa é caracterizada por uma restrição alimentar autoimposta, a fim de obter acentuada perda de peso, mantendo o peso corporal em limites inferiores ao adequado para idade e altura ${ }^{6}$. Além da restrição alimentar, estão presentes o medo de engordar, a preocupação excessiva com o peso e a forma do corpo e alteração da imagem corporal ${ }^{5}$.

A bulimia nervosa é um transtorno alimentar caracterizado por episódios de ingestão compulsiva de alimentos, acompanhado da sensação de perda de controle, culpa e vergonha ${ }^{5}$. Diferentemente dos indivíduos anoréxicos, os bulímicos possuem peso normal ou leve sobrepeso ${ }^{5}$, porém querem desesperadamente emagrecer, e para isso fazem uso de métodos compensatórios como vômitos, laxantes, diuréticos e outros medicamentos?.

Os TA são mais incidentes em praticantes de esportes que preconizam o baixo peso corporal, como dança, corrida de longa distância, natação, ginástica, patinação artística, dentre outros ${ }^{8}$. 0 balé clássico é uma modalidade de dança que valoriza a magreza e a estética corporal, e, desta forma, impulsiona seus praticantes à busca por um corpo ideal, suscitando a insatisfação com a imagem corporal ${ }^{9}$. Aliado a tais fatos, o padrão de beleza imposto pela sociedade moderna ${ }^{10}$, a busca por uma melhor performance ${ }^{11}$, e as pressões por parte dos instrutores, familiares ${ }^{7}$, tornam os bailarinos um grupo de risco para o desenvolvimento dos $\mathrm{TA}^{11,12}$.

No entanto, grupos de bailarinos adolescentes são pouco estudados no que se refere as questões inerentes a transtornos alimentares e insatisfação com a imagem corporal. Portanto, a investigação desses indivíduos é de extrema relevância, haja vista que apresentam vulneravebilidade para o desenvolvimento desses distúrbios.

Neste sentido, o presente estudo teve com objetivo determinar a prevalência de comportamentos de risco para transtornos alimentares e insatisfação com a imagem corporal em bailarinos adolescentes na cidade de São Luís, Maranhão, Brasil.

\section{MÉTODOS}

Trata-se de um estudo transversal realizado com bailarinos clássicos, não profissionais, de ambos os sexos, com idade de 15 a 19 anos, de escolas de balé do município de São Luís, MA, Brasil.

Das quatro maiores escolas de balé existentes na cidade de São Luís, três aceitaram participar deste estudo. A amostra compreendeu 30 bailarinos cujo critério de inclusão foi possuir idade entre 15 a 19 anos, e de exclusão bailarinos que consumiam bebidas alcoólicas habitualmente, diabéticos e bailarinas grávidas. Todos os bailarinos convidados aceitaram participar do estudo.

A coleta de dados foi realizada nas escolas de balé, durante o mês de outubro de 2011, utilizando-se um instrumento estruturado, autoaplicável, com perguntas referentes a dados demográficos e socioeconômicos (Critério de Classificação Econômica Brasil) ${ }^{13}$, registro de rotina de treinamento e dados antropométricos (peso e altura). Para triagem de comportamentos alimentares anormais foram utilizados os questionários EAT-26 (Eating Attitudes Test - Teste de Atitudes Alimentares) ${ }^{14} \mathrm{e}$ BITE (Bulimic Investigatory Test Edinburgh - Teste de Investigação Bulímica de Edinburgh) ${ }^{15}$, e o BSQ (Body Shape Questionnaire - Questionário de Imagem Corporal) para insatisfação da imagem corporal ${ }^{16}$.

O EAT-26 foi utilizado para avaliar a prevalência de comportamento de risco para anorexia nervosa Este instrumento tem sido amplamente utilizado em estudos epidemiológicos para rastrear indivíduos supostamente suscetíveis ao desenvolvimento de distúrbios da conduta alimentar ${ }^{14}$. Consta de 26 questões do tipo escala de Likert, com seis alternativas de resposta, que pontuam de 0 a 3 (sempre=3; muito frequentemente $=2$; frequentemente $=1$; as vezes $=0$; raramente $=0$; nun$\mathrm{ca}=0)$, agrupadas da seguinte forma:

Fator 1: Dieta - as questões refletem recusa patológica às comidas de alto teor calórico e preocupações com a forma física;

Fator 2: Bulimia nervosa - refletem pensamentos sobre comida e atitudes bulímicas;

Fator 3: Controle oral - refletem o autocontrole em relação a comida e reconhecem pressões sociais no ambiente para ganhar peso.

Aplicado o instrumento, os escores obtidos em cada questão do EAT-26 foram somados e computados para cada pessoa avaliada. Os bailarinos que somaram 20 pontos ou mais no EAT-26 foram classificados com comportamento de risco para anorexia nervosa (EAT+), conforme proposto por autores brasileiros ${ }^{2,17}$. Aqueles que não atingiram 20 pontos foram categorizados como EAT negativo (EAT-) ou ausência de anorexia nervosa.

Para avaliar a presença de comportamento de risco para bulimia nervosa e sua intensidade foi utilizado o teste BITE. Este instrumento permite medir episódios bulímicos e fatores ligados à cognição e ao comportamento do indivíduo, podendo ser usado tanto como um instrumento epidemiológico para a identificação de casos subclínicos e clínicos de bulimia, como também para monitorar a evolução dos pacientes e medir a resposta ao tratamento instalado ${ }^{15}$. É composto de 33 questões, onde as de número 1, 13, 21, 23 e 31 recebem um 
(1) ponto para a resposta "não", e as outras vinte e cinco (25) questões recebem um (1) ponto para a resposta "sim". Apresenta duas subescalas, uma voltada para sintomas e outra para a gravidade destes. A escala de sintomas oferece três grupos de escores, conforme recomendado por Cordás e Hochgraf ${ }^{18}$.

Alto (20 pontos ou mais): é considerado presença de comportamento alimentar compulsivo e grande possibilidade de preencher critérios diagnósticos para bulimia nervosa;

Médio (10 a 19 pontos): sugere padrão alimentar não usual, em geral não estão presentes todos os critérios para bulimia. Pessoas com resultados entre 15 e 19 podem representar tanto um grupo subclínico de indivíduos com alimentação compulsiva, como indivíduos bulímicos em estágio inicial ou em recuperação;

Baixo (abaixo de 10 pontos): dentro dos limites da normalidade.

Para análise estatística considerou-se o desfecho dicotômico, no qual foram considerados BITE positivo (BITE + ) aqueles que se inseriram nas categorias alto e médio na escala de sintomas, sendo considerados com comportamento de risco para bulimia, e BITE negativo (BITE-) os que estavam inseridos na faixa de normalidade, conforme proposto no estudo de Magalhães e Mendonça ${ }^{19}$.

A outra escala utilizada do BITE, a escala de gravidade, é constituída pelos itens 6, 7 e 27 do questionário, e mede a gravidade do comportamento compulsivo por meio da frequência de atitudes, ramificando-se em três estágios ${ }^{18}$ :

Alto (escore $\geq 10$ pontos): indicam alto grau de gravidade, podendo apontar a presença de vômito psicogênico ou abuso de laxante sem comportamento compulsivo;

Moderado (entre 5 e 9 pontos): são considerados clinicamente significativos;

Baixo (até 5 pontos): resultado clinicamente não significativo.

O BSQ foi utilizado para avaliar a insatisfação com a imagem, que avalia as preocupações com a forma do corpo, autodepreciação devido à aparência e a sensação de estar gordo ${ }^{17}$. O questionário distingue dois aspectos específicos da imagem corporal: a exatidão da estimativa do tamanho corporal e os sentimentos em relação ao corpo (insatisfação ou desvalorização da forma física) ${ }^{20}$. Trata-se de um questionário autoaplicável do tipo escala de Likert, composto por 34 questões, com seis possibilidades de respostas, que pontuam de 1 a 6 pontos (sempre $=6$; muito frequentemente $=5$; frequentemente $=4$; às vezes $=3$; raramente $=2$; nunca $=1)^{16}$.

O resultado do teste BSQ é a somatória dos 34 itens contidos no questionário e sua classificação reflete os níveis de preocupação com a imagem corporal, tais como: ausência de insatisfação com a imagem corporal (0-80 pontos); leve insatisfação com a imagem corporal (81110 pontos); moderada insatisfação com a imagem corporal (111-140 pontos); grave insatisfação com a imagem corporal (< 140 pontos $)^{16}$.

Para avaliar o estado nutricional dos bailarinos foi utilizado o índice de massa corporal (IMC) ${ }^{21}$, obtido por meio da relação entre o peso $(\mathrm{kg})$ e o quadrado da altura $\left(\mathrm{m}^{2}\right)$, cujos dados foram autoreferidos. Estudos indicam que a correspondência entre o peso e altura referidos e aferidos é satisfatória, podendo ser utilizado em pesquisas populacionais quando a aferição for inviável ou quando houver necessidade de tornar o estudo mais rápido ou diminuir seu custo ${ }^{22}$. A classificação do IMC para idade utilizada foi a proposta pela Organização Mundial de Saúde (magreza < percentil 3; eutrofia $\geq$ percentil 3 e < percentil 85; e excesso de peso $\geq$ percentil 85$)^{23}$.

Para análise estatística, as variáveis quantitativas foram representadas por meio de média e desvio padrão ou mediana e valores mínimo e máximo, e as qualitativas, por frequências simples e percentuais. A normalidade foi testada pelo teste de Shapiro-Wilk e o teste Qui-quadrado ou Exato de Fischer foi utilizado para analisar a associação entre as variáveis qualitativas. O nível de significância adotado foi de $5 \%$ e os dados foram analisados no programa estatístico STATA 10.0.

Este estudo foi aprovado pelo Comitê de Ética em Pesquisa da Universidade Federal do Maranhão, Brasil (Parecer Consubstanciado No 23115008665/2011-31), e todos os bailarinos participantes do estudo obtiveram, dos pais ou responsáveis, a assinatura do Termo de Consentimento Livre e Esclarecido.

\section{RESULTADOS}

Dos 30 bailarinos avaliados, 70\% eram meninas, idade média de 16,8 \pm 1,4 anos, variando entre 15 a 19 anos. Segundo o Critério de Classificação Econômica Brasil (CCEB), metade da amostra estudada pertencia à classe econômica C e 36,7\% às classes D/E. A avaliação do estado nutricional, pelo IMC, revelou a grande maioria eutrófica (96,7\%), mediana 19,2 kg/m², variando de 16,2 a $26,4 \mathrm{~kg} / \mathrm{m}^{2}$ (tabela 1). O tempo de prática de balé foi de 3,2 $\pm 2,5$ anos.

A prevalência de comportamento de risco para TA foi de 30,0\% para risco de anorexia nervosa e 40,0\% para risco de bulimia nervosa (escala de sintomas com escore alto e médio). Analisando a escala de gravidade do BITE, 10,0\% dos bailarinos estavam inseridos na faixa classificada como moderada, sendo esta considerada clinicamente significativa para bulimia nervosa. O BSQ identificou 26,7\% dos investigados com algum grau de insatisfação com a imagem corporal. O uso de métodos compensatórias para perda de peso foi relatado por 33,7\% do grupo. Analisando o comportamento de risco para TA, insatisfação com a imagem corporal e a utilização de métodos compensatórios por sexos, não foi observada diferença estatisticamente significante ( $p>0,05)$ (tabela 2).

Vale salientar que, das práticas compensatórias utilizadas, o jejum por um dia inteiro foi o mais citado pelos bailarinos (33,3\%), seguido do uso de métodos purgativos (16,7\%) (tabela 3).

\section{DISCUSSÃO}

A prevalência de comportamento de risco para anorexia nervosa observada nesta amostra (30,0\%) foi maior do que a encontrada em investigação realizada em Rondônia (8,3\%) com 12 bailarinas de 12 a 19 anos $^{24}$. Alguns estudiosos apontam que a taxa de incidência de anorexia nervosa entre 15 e 19 anos aumentou significativamente ${ }^{25}$, o que poderia justificar a elevada freqüência observada, haja vista que a faixa etária coincide com a dos bailarinos investigados.

No Rio de Janeiro, Ribeiro e Veiga ${ }^{26}$, avaliando bailarinos profissionais, de ambos os sexos, também identificaram uma prevalência inferior $(11,5 \%)$ a do presente estudo para anorexia nervosa, provavelmente devido à maior média de idade dos bailarinos cariocas (36,8 \pm 10,5 anos).

Dos estudos internacionais realizados na última década, a maior prevalência de comportamento de risco para anorexia nervosa encontrada (18,5\%) foi em uma investigação realizada na República Tcheca

Tabela 1. Características sociodemográficas e estado nutricional de bailarinos adolescentes da cidade de São Luís, Maranhão, 2011.

\begin{tabular}{c|c|c}
\hline Variáveis de estudo & $\mathbf{n}$ & $\%$ \\
\hline Sexo & & \\
\hline Feminino & 21 & 70,0 \\
\hline Masculino & 9 & 30,0 \\
\hline Classes socioeconômicas (CCEB) & & 13,3 \\
\hline Classe A-B & 4 & 50,0 \\
\hline Classe C & 15 & 36,7 \\
\hline Classe D-E & 11 & \\
\hline Estado nutricional (IMC) & & 96,7 \\
\hline Eutrofia & 29 & 3,3 \\
\hline Sobrepeso/Obesidade & 1 &
\end{tabular}

CCEB - Critério Classificação Econômica Brasil; IMC - Índice de Massa Corporal. 
Tabela 2. Prevalência de comportamento de risco para transtornos alimentares (EAT-26 e BITE), insatisfação com a imagem (BSQ) e utilização de métodos compensatórios por sexo, em bailarinos adolescentes da cidade de São Luís, Maranhão, 2011.

\begin{tabular}{|c|c|c|c|c|c|c|c|}
\hline \multirow{2}{*}{ Instrumentos } & \multicolumn{2}{|c|}{ Total $(n=30)$} & \multicolumn{2}{|c|}{ Feminino $(n=21$} & \multicolumn{2}{|c|}{ Masculino $(n=9)$} & \multirow{2}{*}{$p$-valor } \\
\hline & $n$ & $\%$ & $n$ & $\%$ & $n$ & $\%$ & \\
\hline \multicolumn{7}{|c|}{ EAT } & 0,794 \\
\hline Positivo & 9 & 30,0 & 6 & 28,6 & 3 & 33,3 & \\
\hline Negativo & 21 & 70,0 & 15 & 71,4 & 6 & 66,7 & \\
\hline Total & 30 & 100,0 & 21 & 100,0 & 9 & 100,0 & \\
\hline \multicolumn{7}{|c|}{ BITE: Escala de sintomas } & 0,626 \\
\hline Positivo & 12 & 40,0 & 9 & 42,8 & 3 & 33,3 & \\
\hline Negativo & 18 & 60,0 & 12 & 57,2 & 6 & 66,7 & \\
\hline Total & 30 & 100,0 & 21 & 100,0 & 9 & 100,0 & \\
\hline \multicolumn{7}{|c|}{ BITE: Escala de gravidade } & 0,328 \\
\hline $\begin{array}{c}\text { Alto grau de } \\
\text { gravidade }\end{array}$ & 0 & 0,0 & 0 & 0,0 & 0 & 0,0 & \\
\hline $\begin{array}{c}\text { Moderado } \\
\text { (clinicamente } \\
\text { significativo) } \\
\end{array}$ & 3 & 10,0 & 3 & 14,3 & 0 & 0,0 & \\
\hline $\begin{array}{c}\text { Baixo } \\
\text { (clinicamente não } \\
\text { significativo) } \\
\end{array}$ & 27 & 90,0 & 18 & 85,7 & 9 & 100,0 & \\
\hline Total & 30 & 100,0 & 21 & 100,0 & 9 & 100,0 & \\
\hline \multicolumn{7}{|c|}{ BSQ } & 0,762 \\
\hline $\begin{array}{c}\text { Grave } \\
\text { insatisfaçãa com } \\
\text { imagem corporal }\end{array}$ & 1 & 3,3 & 1 & 4,8 & 0 & 0,0 & \\
\hline $\begin{array}{c}\text { Moderada } \\
\text { insatisfação com a } \\
\text { imagem corporal }\end{array}$ & 2 & 6,7 & 2 & 9,5 & 0 & 0,0 & \\
\hline $\begin{array}{c}\text { Leve } \\
\text { insatisfação com a } \\
\text { imagem corporal }\end{array}$ & 5 & 16,7 & 4 & 19,0 & 1 & 11,1 & \\
\hline $\begin{array}{c}\text { Ausência de } \\
\text { insatisfação com a } \\
\text { imagem corporal }\end{array}$ & 22 & 73,3 & 14 & 66,7 & 8 & 88,9 & \\
\hline Total & 30 & 100,0 & 21 & 100,0 & 9 & 100,0 & \\
\hline \multicolumn{7}{|c|}{ Métodos compensatórios } & 0,804 \\
\hline Não utilizam & 19 & 63,3 & 13 & 61,9 & 6 & 66,7 & \\
\hline Utilizam & 11 & 33,7 & 8 & 38,1 & 3 & 33,3 & \\
\hline Total & 30 & 100,0 & 21 & 100,0 & 9 & 100,0 & \\
\hline
\end{tabular}

EAT - Teste de Atitudes Alimentares; BITE - Teste de Investigação Bulímica de Edinburgh; BSQ - Teste de imagem corporal.

Tabela 3. Frequência de métodos compensatórios utilizados para auxiliar na perda de peso em bailarinos adolescentes da cidade de São Luís, Maranhão, 2011.

\begin{tabular}{c|c|c}
\hline Condutas & $\mathbf{n}$ & $\%$ \\
\hline Jejum por um dia inteiro & 10 & 33,3 \\
\hline Laxante & 3 & 10,0 \\
\hline $\begin{array}{c}\text { Comprimido para } \\
\text { emagrecer }\end{array}$ & 1 & 3,3 \\
\hline Vômito & 1 & 3,3 \\
\hline Diurético & 0 & 0,0 \\
\hline
\end{tabular}

com um grupo de dançarinos ${ }^{10}$. Essa investigação utilizou o EAT-40, escala que originou o EAT-26, o qual mantem uma forte correlação com as variáveis clínicas e psicométricas, permitindo comparação com os resultados desta pesquisa ${ }^{14}$.

Analisando o comportamento de risco para bulimia nervosa, considerando a escala de sintomas do instrumento BITE, observou-se 40,0\% dos bailarinos com este comportamento. Esta prevalência é superior a encontrada (29,5\%) por Ribeiro e Veiga ${ }^{26}$, que investigaram bailarinos profissionais com média de idade 36,8 + 10,5 anos. Uma possível explicação para a elevada prevalência revelada neste estudo é o fato de a bulimia nervosa iniciar-se no final da adolescência e início da idade adulta³.
Por outro lado, um estudo com 110 bailarinas italianas, entre 12 e 30 anos, identificou uma frequência de bulimia nervosa de apenas $1,8 \%{ }^{27}$. É importante ressaltar que a divergência entre a prevalência de comportamento de risco para transtornos alimentares encontrada nessa investigação e os demais da literatura, pode ser devido a diversos fatores como utilização de diferentes instrumentos de avaliação, diferenças nas características da amostra estudada (tamanho e faixa etária), ou pode ser reflexo de uma tendência no desenvolvimento precoce de transtornos alimentares nos últimos anos, em que tem se observado aumento do número de casos em idades mais novas ${ }^{28}$.

Além disso, como a identificação da presença de comportamentos de risco para TA, por meio de questionários, não garante o diagnóstico, supõem-se que esses adolescentes estão em uma fase de maior exposição aos fatores de risco para o desenvolvimento destes transtornos, como a preocupação com a imagem corporal e atitudes para redução de peso ${ }^{28}$

Apesar da literatura relatar que a prevalência de TA é maior entre as mulheres, por serem mais vulneráveis às pressões socioculturais, econômicas e estéticas, constituindo um grupo de maior risco para o desenvolvimento de distúrbios alimentares ${ }^{22}$, este estudo não revelou diferença estatisticamente significante entre os sexos. Em relação a este aspecto, as pesquisas apontam para um aumento na prevalência de TA nos homens ${ }^{17}$, o que parece ser devido à crescente preocupação com aparência física oriunda dos novos padrões estéticos criados para este gênero ${ }^{29}$.

Vale salientar que, embora quase a totalidade dos bailarinos se apresente eutrófica, 26,7\% estavam insatisfeitos com sua imagem corporal (BSQ), indicando o quanto é comum em virtude do atual culto a magreza, que os indivíduos mesmo eutróficos, se sintam insatisfeitos com seus $\operatorname{corpos}^{26}$. A insatisfação da imagem corporal não apresentou diferenças entre os sexos, apontando que ambos possuem uma preocupação excessiva com o corpo, em decorrência do padrão de magreza exigido no balé12,26.

Esta insatisfação da imagem corporal observada é um resultado preocupante, haja vista que a insatisfação com o corpo está associada a práticas inadequadas para perda de peso, como indução ao vômito, jejum e laxantes, etc. . . Ademais, é bem estabelecido que a insatisfação com a imagem corporal é considerada um dos principais sintomas dos transtornos alimentares, caracterizado pela auto-avaliação negativa do indivíduo em relação ao seu peso e forma corporal ${ }^{9}$, representando fatores de risco para o aumento da prevalência dos TA.

Apesar da escala de sintomas do BITE ter revelado 40,0\% dos bailarinos com comportamento de risco para bulimia nervosa, a aplicação da escala de gravidade deste instrumento, inversamente, revelou 90\% dos bailarinos inseridos no score clinicamente não significativo para TA. Embora não apontando gravidade em sua maioria, esta é uma condição preocupante, pois há possibilidade de evolução dos casos de moderada gravidade para comportamentos de alta gravidade e para a instalação dos TA.

Consoante os resultados apresentados, ressalta-se que o presente estudo se baseou exclusivamente em questionários de autopreenchimento (EAT, BITE e BSQ), que são instrumentos alternativos na investigação epidemiológica de comportamentos de risco de $\mathrm{TA}^{30}$, contudo, não são suficientes para se estabelecer diagnóstico de anorexia ou bulimia nervosa, já que são necessárias entrevistas clínicas para confirmação dos resultados ${ }^{3}$. Salienta-se também que o tamanho da amostra dificultou a confirmação de algumas tendências de associações entre as variáveis investigadas.

Os resultados desta pesquisa demonstram que os bailarinos apresentaram alta prevalência de comportamentos alimentares de risco, pois estão inseridos em uma modalidade desportiva que preconiza o baixo peso corporal e supervaloriza a estética. 
O estudo apontou que mesmo apresentando estado nutricional adequado, uma parcela significativa dos bailarinos adolescentes avaliados apresentam insatisfação com a imagem corporal e comportamentos de risco para TA. Tais achados sugerem a preponderância do padrão estético em detrimento da saúde, e que a prática do balé parece ser um fator primordial para uma possível susceptibilidade desses bailarinos ao desenvolvimento de TA.

É necessário o desenvolvimento de outras pesquisas com esse grupo, já que existem poucos estudos que relacionam os transtornos alimentares e a dança, com o objetivo de conhecer fatores de proteção ou de risco para instalação desses TA na população estudada.

\section{CONCLUSÃO}

Assim, é importante que nutricionistas atuem junto a este segmento, visando à prevenção ou detecção precoce da presença de transtornos de conduta alimentar, o combate a práticas alimentares inadequadas, afim de permitir crescimento harmonioso e saudável para os bailarinos adolescentes, além de melhorar o desempenho atlético, sem prejuízo para a sua saúde.

Todos os autores declararam não haver qualquer potencial conflito de interesses referente a este artigo.

\section{REFERÊNCIAS}

1. Ricca V, Rotella F, Mannucci E, Ravaldi C, Castellini G, Lapi F, et al. Eating behaviour and body satisfaction in Mediterranean children: the role of the parents. Clin Pract Epidemiol Ment Health. 2010;6:59-65.

2. Laus MF, Moreira RCM, Costa TMB. Diferenças na percepção da imagem corporal, no comportamento alimentar e no estado nutricional de universitárias das áreas de saúde e humanas. Rev Psiquiatr Rio Gd Sul. 2009;31(3):192-6.

3. American Dietetic Association. Position of the American Dietetic Association: Nutrition intervention in the treatment of anorexia nervosa, bulimia nervosa, and eating disorders not otherwise specified (EDNOS). J Am Diet Assoc. 2001;101(7):810-9.

4. Morgan CM, Vecchiatti IR, Negrão AB. Etiologia dos transtornos alimentares: aspectos biológicos, psicológicos e sócio-culturais. Rev Bras Psiquiatr. 2002; 24(Suppl 3):18-23.

5. American Psychiatric Association. Diagnostic and statistical manual of mental disorder. 4th ed. Washington: American Psychiatric Press; 1994

6. Organização Mundial De Saúde. Classificação dos transtornos mentais e de comportamentos da CID-10: descrições clínicas e diretrizes diagnósticas. Tradução de Dorgival Caetano. Porto Alegre: Artes Médicas; 1993.

7. Oliveira FP de, Bosi MLG, Vigário PS, Viera RS. Comportamento alimentar e imagem corporal em atletas. Rev Bras Med Esporte. 2003;9(6):348-356

8. Monthuy-Blanc J, Maiano C, Therme P. Prevalence of eating disorders symptoms in nonelite ballet dancers and basketball players: an exploratory and controlled study among French adolescent girls. Rev Epidemiol Sante Publique. 2010;58(6):415-24.

9. Hass AN, Garcia ACD, Bertoletti J. Imagem corporal e bailarinas profissionais. Rev Bras Med Esporte. 2010;16(3):182-185.

10. Janout $V$, Janoutova G. Eating disorders risk groups in the Czech Republic — cross-sectional epidemiologic pilot study. Biomed Pap Med Fac Univ Palacky Olomouc Czech Repub. 2004;148(2):189-93.

11. Herbrich L, Pfeiffer E, Lehmkuhl U, Schneider N. Anorexia athletic in pre-professional ballet dancers. J Sports Sci. 2011;29(11):1115-23

12. Özgen $L$, Kisaç I. Drive for thinness, bulimia and body dissatisfaction in Turkish ballet dancers and ballerinas. Proc Social Behav Sci. 2009;1(1):2218-21

13. Associação Brasileira de Empresas de Pesquisas. Critério de Classificação Econômica Brasil. 2011. Disponível em: <http://www.abep.org/ codigosguias/ABEP_CCEB.pdf>. (Acessado em: 18 jan de 2011).

14. Garner DM, Olmsted MP, Boher Y, Garfikel PE. The Eating Attitudes Test: psychometric features and clinical correlates. Physiol Med. 1982;(12):871-8.

15. Henderson M, Freeman CPL. A self-rating scale for bulimia - The "BITE". Br J Psychiatry. 1987;150:18-24.

16. Cooper P, Taylor MJ, Cooper Z, Fairburn CG. The development and validation of the Body Shape Questionnaire. Int J Eat Disord. 1987;6:485-94.
17. Vilela JEM, Lamounier JA, Filho MAD, Neto JRB, Horta GM.Transtornos alimentares em escolares. Pediatr. 2004;80(1):49- 54

18. Cordás TA, Hochgraf PB. O "BITE": instrumento para avaliação da bulimia nervosa versão para o português. J Bras Psiquiatr. 1993;42(3):141-4

19. Magalhães VC, Medonça GAS. Transtornos alimentares em universitárias estudo de confiabilidade da versão brasileira de questionários autopreenchíveis. Rev Bras Epidemiol. 2005;8(3):236-45.

20. Cordás TA, Neves JEP. Escalas de avaliação de transtornos alimentares. Rev Psiquiatr Clin. 1999;26(1):41-8.

21. World Health Organization [Internet]. Physical status: the use and interpretation of anthropometry. Report of a WHO Expert Committee. World Health Organ Tech Rep. Geneva: WHO 1995 [cited 2011 jul 18]. Disponível em: <http://www.who.int/childgrowth/publications/physical_status/en/index. html>[http://whqlibdoc.who.int/trs/WHO_TRS_854.pdf].

22. Bosi MLM, Luiz RR, Morgado CMC, Costa MLS, Carvalho RJ. Autopercepção da imagem corporal entre estudantes de nutrição: um estudo no município do Rio de Janeiro. J Bras Psiquiatr. 2006;55(2):108-13.

23. World Health Organization [Internet]. Growth reference data for 5-19 years [Internet]. Geneva: WHO 2007 [cited 2011 jul 18]. Disponivel em: <http://www.who.int/growthref/who2007_bmi_for_age/ en/index.html>[http://www.who.int/growthref/en/]

24. Pereira IO, Lima AP, Cardoso F, Oliveira GL, Filho JF, Perini TA. Distorção da imagem corporal e tríade da mulher atleta em bailarinas clássicas. Revista Digital [internet]. 2010. Disponível em: <http://www.efdeportes. com/efd149/triade-da-mulher-atleta-em-bailarinas.classicas.htm. (Acessado em 25 de março de 2011).

25. Yang SJ, Kim JM, Yonn S. Disturbed eating attitudes and behaviors in South Korean boys and girls: a school-based cross-sectional study. Yonsei Med J. 2010;51(3) 302-9.

26. Ribeiro LG, Veiga GV. Imagem corporal e comportamentos de risco para transtornos alimentares em bailarinos profissionais. Rev Bras Med Esporte. 2010;16(2): 99-102.

27. Ravaldi C, Vannacci A, Bolognesi E, Mancini S, Faravelli C, Ricca V. Gender role, eating disorder symptoms, and body image concern in ballet dancers. J Psychosom Res. 2006;61(4):529-35.

28. Alves E, Vasconcelos FAG, Calvo MCM, Neves J. Prevalência de sintomas de anorexia nervosa e insatisfação com a imagem corporal em adolescentes do sexo feminino do Município de Florianópolis, Santa Catarina, Brasil. Cad Saúde Pública. 2008;24(3):503-12.

29. Carvalho PVLC. Anorexia e bulimia em adolescentes [dissertação]. São Luís: Universidade Federal do Maranhão; 2008

30. Ferreira JES, Veiga GV. Confiabilidade (teste-reteste) de um questionário simplificado para triagem de adolescentes com comportamentos de risco para transtornos alimentares em estudos epidemiológicos. Rev Bras Epidemiol. 2008;11(3):393-401. 